\title{
A model of repression: CTD analogs and PIE-1 inhibit transcriptional elongation by P-TEFb
}

\author{
Fan Zhang, ${ }^{1,4}$ Matjaz Barboric, ${ }^{1,2,4}$ T. Keith Blackwell, ${ }^{3}$ and B. Matija Peterlin ${ }^{1,5}$ \\ ${ }^{1}$ Departments of Medicine, Microbiology and Immunology, Rosalind Russell Medical Research Center, University of \\ California at San Francisco, San Francisco, California 94143, USA; ${ }^{2}$ Institute of Biochemistry, Medical Faculty of the \\ University of Ljubljana, Republic of Slovenia; ${ }^{3}$ Center for Blood Research and Department of Pathology, Harvard Medical \\ School, Boston, Massachusetts 02115, USA
}

\begin{abstract}
The positive transcription elongation factor $\mathrm{b}(\mathrm{P}-\mathrm{TEFb})$ contains cyclin $\mathrm{T} 1$ (CycT1) and cyclin-dependent kinase 9 (Cdk9). For activating the expression of eukaryotic genes, the histidine-rich sequence in CycT1 binds the heptapeptide repeats in the C-terminal domain (CTD) of RNA polymerase II (RNAPII), whereupon Cdk9 phosphorylates the CTD. We found that alanine-substituted heptapeptide repeats that cannot be phosphorylated also bind CycT1. When placed near transcription units, these CTD analogs block effects of P-TEFb. Remarkably, the transcriptional repressor PIE-1 from Caenorhabditis elegans behaves analogously. It binds CycT1 via an alanine-containing heptapeptide repeat and inhibits transcriptional elongation. Thus, our findings reveal a new mechanism by which repressors inhibit eukaryotic transcription.
\end{abstract}

[Keywords: P-TEFb; CTD; PIE-1; transcription; repression; elongation]

Received December 17, 2002; revised version accepted January 27, 2003.

Regulation of eukaryotic transcription operates at many levels, which include dynamic changes in chromatin and effects on RNA polymerase II (RNAPII). For example, by recruiting ATP-dependent remodeling complexes (SWI/ SNF, 1sw2p, Mi-2, etc.; Goldmark et al. 2000; Langst and Becker 2001), histone acetyltransferases (HATs) and histone deacetylases (HDACs; Struhl 1998; Khochbin et al. 2001), activators and repressors influence the opening and closing of chromatin, respectively. Open chromatin favors the assembly of the preinitiation complex (PIC) on promoters. There, general transcriptional factors (GTFs) and the Mediator position RNAPII correctly at the beginning of genes (Zawel and Reinberg 1995; Lemon and Tjian 2000). Sequence-specific activators on distal enhancers then potentiate these effects and increase rates of transcriptional elongation (Taube et al. 2002). Finally, boundary elements enclose distinct transcription units and give them autonomy (Blackwood and Kadonaga 1998).

The evolutionary conserved C-terminal domain (CTD) of RNAPII is a key player in transcription (Bartolomei et al. 1988; Prelich 2002). Its main function is to coordinate steps in transcription and cotranscriptional pre-mRNA processing. Depending on the phosphorylation status of

\footnotetext{
${ }^{4}$ These authors contributed equally to this work.

${ }^{5}$ Correponding author.

E-MAIL matija@itsa.ucsf.edu; FAX (415) 502-1901.

Article published online ahead of print. Article and publication date are at http://www.genesdev.org/cgi/doi/10.1101/gad.1068203.
}

serines at positions 2 and 5 of the 52 repeats of its YSPTSPS heptapeptide, the CTD is a platform for the ordered assembly of different transcriptional machineries. First, the unphosphorylated CTD forms PIC with GTFs and the Mediator at the promoter (Orphanides et al. 1996; Myers and Kornberg 2000). Next, during promoter clearance, Cdk7 of TFIIH phosphorylates the serine at position 5 in the CTD (Lu et al. 1992), which recruits and activates capping enzymes (Guzman and Lis 1999; Ho and Shuman 1999). In addition, this RNAPII complex associates with the negative transcription elongation factor (N-TEF; Price 2000), which most likely consists of the 5,6-dichloro-1-beta-D-ribofuranosylbenzimidazole (DRB)-sensitivity inducing factor (DSIF; Wada et al. 1998) and the negative elongation factor (NELF; Yamaguchi et al. 1999), thus arresting transcription. Finally, P-TEFb is recruited to overcome these blocks (Price 2000). It phosphorylates preferentially the serine at position 2 in the CTD (Zhou et al. 2000; Shim et al. 2002), which now binds proteins that are involved in splicing and polyadenylation (Mortillaro et al. 1996; McCracken et al. 1997; Orphanides and Reinberg 2002), and the suppressor of Ty protein 5 (Spt5) in DSIF (Ivanov et al. 2000). By modifying RNAPII and neutralizing N-TEF, $\mathrm{P}-\mathrm{TEFb}$ increases rates of elongation of transcription. Consistent with this mechanism, the phosphorylated serines at position 2 in the CTD are found with RNAPII on actively transcribed genes (Komarnitsky et al. 2000). When transcription terminates, the CTD is dephos- 
phorylated, so that RNAPII is able to repeat this transcription cycle (Dahmus 1996; Cho et al. 1999).

$\mathrm{P}-\mathrm{TEFb}$ consists of a catalytic subunit (Cdk9) and a regulatory subunit, which can be CycT1, CycT2a, CycT2b, or CycK (Peng et al. 1998; Price 2000). CycT1 is required for HIV replication, in which the viral transactivator Tat recruits P-TEFb to the transactivation response (TAR) RNA structure at the 5' end of all transcripts (Garber et al. 2000). In this way, Cdk9 phosphorylates the CTD and Spt5 to synthesize full-length viral transcripts (Zhou et al. 2000). Notably, DRB and flavopiridol, which represent two specific inhibitors of Cdk9, block HIV replication in infected cells (Marshall et al. 1996; Chao et al. 2000). Beside Tat, other eukaryotic transcriptional activators also bind P-TEFb to stimulate transcriptional elongation by RNAPII. These include CIITA (Kanazawa et al. 2000), NF-кB (Barboric et al. 2001), c-Myc (Eberhardy and Farnham 2002), and the androgen receptor (Lee et al. 2001). In the case of RelA and CIITA, they bind the N-terminal cyclin boxes in CycT1. A histidine-rich sequence in the $\mathrm{C}$ terminus of CycT1 then binds the unphosphorylated CTD. When P-TEFb is placed $5^{\prime}$ to promoters and $3^{\prime}$ to coding sequences, this histidine-rich sequence is all that is required to recognize and target Cdk9 to the unphosphorylated CTD of RNAPII at promoters (Taube et al. 2002).

In this scheme, some repressors could inhibit transcription by targeting P-TEFb. Simply by diverting the histidine-rich stretch in CycT1 away from the CTD of RNAPII, they could block transcriptional elongation. Thus, the ideal repressor of this type should possess one or more CTD-like repeats, which could not be phosphorylated. Pharynx and intestine in excess protein 1 (PIE-1), which represses transcription globally in early embryonic germ cell precursors in Caenorhabditis elegans (Mello et al. 1992; Seydoux et al. 1996), contains just such a CTD-like heptapeptide repeat, in which the serines at positions 2 and 5 are substituted with alanines (Batchelder et al. 1999). When these alanines are mutated to serines to resemble the wild-type CTD or acidic residues (glutamate and aspartate) to resemble the phosphorylated CTD, PIE-1 only partially or no longer represses transcription. Moreover, in vivo disruption of this CTD-like repeat does not influence the stability or localization of PIE-1, but impairs its function, indicating the importance of this domain for maintaining transcriptional silencing in the germ line.

In this study, we investigated this mechanism of transcriptional repression. First, an artificial alanine-substituted but not glutamate-substituted CTD analog that was tethered to DNA blocked effects of P-TEFb and bound CycT1. Second, PIE-1 behaved identically. Importantly, only PIE-1 with the wild-type but not the glutamate- and aspartate-substituted heptapeptide repeat bound CycTl and inhibited transcriptional elongation. We propose that because neither alanine-substituted CTD nor the heptapeptide repeat in PIE-1 could be phosphorylated, P-TEFb did not dissociate from this repressor and was unable to modify its physiological target, the CTD of RNAPII.

\section{Results}

Wild-type and alanine-substituted, but not glutamate-substituted heptapeptide repeats repress transcriptional activation by $P$-TEFb

To test the hypothesis that different CTD analogs could have distinct effects on transcription mediated by $\mathrm{P}-\mathrm{TEFb}$, we created plasmid effectors and target, which are presented in Figure 1A. The plasmid target pG6L6CAT contained six Gal4 (upstream activator sequences) and LexA (SOS) DNA-binding sites, followed by the HIV-1 promoter and the CAT reporter gene. Plasmid effectors encoded the wild-type CycT1 and Cdk9 proteins fused to the Gal4 DNA-binding domain (DBD; Gal.CycT1 and Gal.Cdk9, respectively) and represented transcriptional activators (Taube et al. 2002). For the putative repressor, we expressed the mutant Lex.CTD(AA)17 fusion protein that cannot be phosphorylated. A further series of tandemly repeated wild-type and mutant heptapeptide repeats fused to the LexA DBD represented important controls. Among them, Lex.CTD(SS)16 contained the wild-type CTD. Mutant Lex.CTD(ES)15 and Lex.CTD(SE)19 fusion proteins mimicked constitutively phosphorylated heptapeptide repeats, respectively.

When we coexpressed Lex fusion proteins with pG6L6CAT in Hela cells, the wild-type Lex.CTD(SS)16 chimera increased CAT activity only ninefold (Fig. 1B, lane 3). Importantly, the other mutant Lex chimeras had no effect (Fig. 1B, lanes 2,4,5). In contrast, Gal.CycT1 and Gal.Cdk9 fusion proteins increased CAT activities 55fold and 45-fold over basal levels, respectively (Fig. 1B, cf. lanes 6 and 7,12 and 13). This activation was inhibited $80 \%$ by the coexpression of equal amounts of the mutant Lex.CTD(AA) 17 fusion protein (Fig. 1B, cf. lanes 7,8 and 13,14). Whereas the mutant Lex.CTD(ES)15 and Lex.CTD(SE)19 fusion proteins had no effect (Fig. 1B, cf. lanes 7,10-12,16,17), the Lex.CTD(SS)16 chimera more than halved the activation by P-TEFb (Fig. 1B, cf. lanes 7,9 and 13,15 ). If one subtracts the positive effects of the wild-type Lex.CTD(SS)16 chimera, then its effects on PTEFb were even greater (Fig. 1B, cf. lanes 3,9,15). The expression of all fusion proteins was comparable (Fig. 1B, bottom). We conclude that the degree of CTD phosphorylation correlates with the inhibition of transcriptional activation by P-TEFb. Thus, whereas the heptapeptide repeats that cannot be phosphorylated act as the strongest repressor, analogs of constitutively phosphorylated CTD have no effect. Importantly, the "pseudosubstrate" of P-TEFb can block its transcriptional effects in cells.

Wild-type and alanine-substituted, but not glutamate-substituted heptapeptide repeats bind CycT1

The binding between the histidine-rich sequence within the C terminus of CycT1 and the CTD of RNAPII is required for $\mathrm{P}-\mathrm{TEFb}$ to activate transcription (Taube et al. 2002). Our observation that CTD analogs affected transcriptional effects of P-TEFb differently, suggested 
Figure 1. Wild-type and alanine-substituted CTD analogs repress transcriptional activation by P-TEFb. (A) Schematic representation of plasmid effectors and target. The plasmid effector Gal.CycT1 directed the expression of the wild-type CycT1 protein from the EFBOS promoter. CycT1 was fused at its $\mathrm{N}$ terminus to the Gal4 DNA-binding domain [DBD; Gal4(1-147), hatched box] and the c-Myc epitope tag (Myc, black box). Also depicted are two cyclin boxes (black box), Tat and TAR recognition motif (TRM; checkered box), coilcoiled region (coil-coil), histidine-rich region (his-rich), and PEST sequence (all in black boxes). The plasmid effector Gal.Cdk9 expressed the wild-type Cdk9 protein (white box) from the SV40 promoter and was fused at its N terminus to Gal4(1-147). Also presented are the plasmid effectors Lex.CTD(SS)16, Lex.CTD(AA)17, Lex.CTD(ES)15, and Lex.CTD(SE)19. They expressed tandemly repeated heptapeptides from the CMV promoter and were fused at their $\mathrm{N}$ termini to the LexA DBD [LexA(1-87), cross-hatched box] and the $\mathrm{X}$-press epitope tag (Xpress, gray box). The cartoons represent heptapeptides; their amino acid sequences and numbers of repeats are depicted to their right. Bold letters within heptapeptides represent wild-type amino acids, whereas underlined bold letters represent the mutated ones. In the cartoons, white balls represent serine residues, balls in balls represent alanine residues, and black balls represent glutamate residues. The arrows indicate the start site of transcription. Encircled pA indicates the polyadenylation site. The plasmid target pG6L6CAT contains six synthetic Gal4 DNA-binding sites (UAS; hatched boxes), which are placed $442 \mathrm{bp}$ upstream of six LexA operator sites (SOS; cross-hatched boxes). PRO represents HIV LTR; it directs the expression of the CAT reporter gene. $(B)$ The effect of CTD analogs on P-TEFb. Hela cells expressed pG6L6CAT (bars 1-17). Proteins that were coexpressed with the plasmid reporter are indicated by plus signs above the CAT data. Expression levels of CycT1, Cdk9, and LexA fusion proteins are presented below the CAT data and are indicated by arrows and curved lines. NS depicts nonspecific proteins.
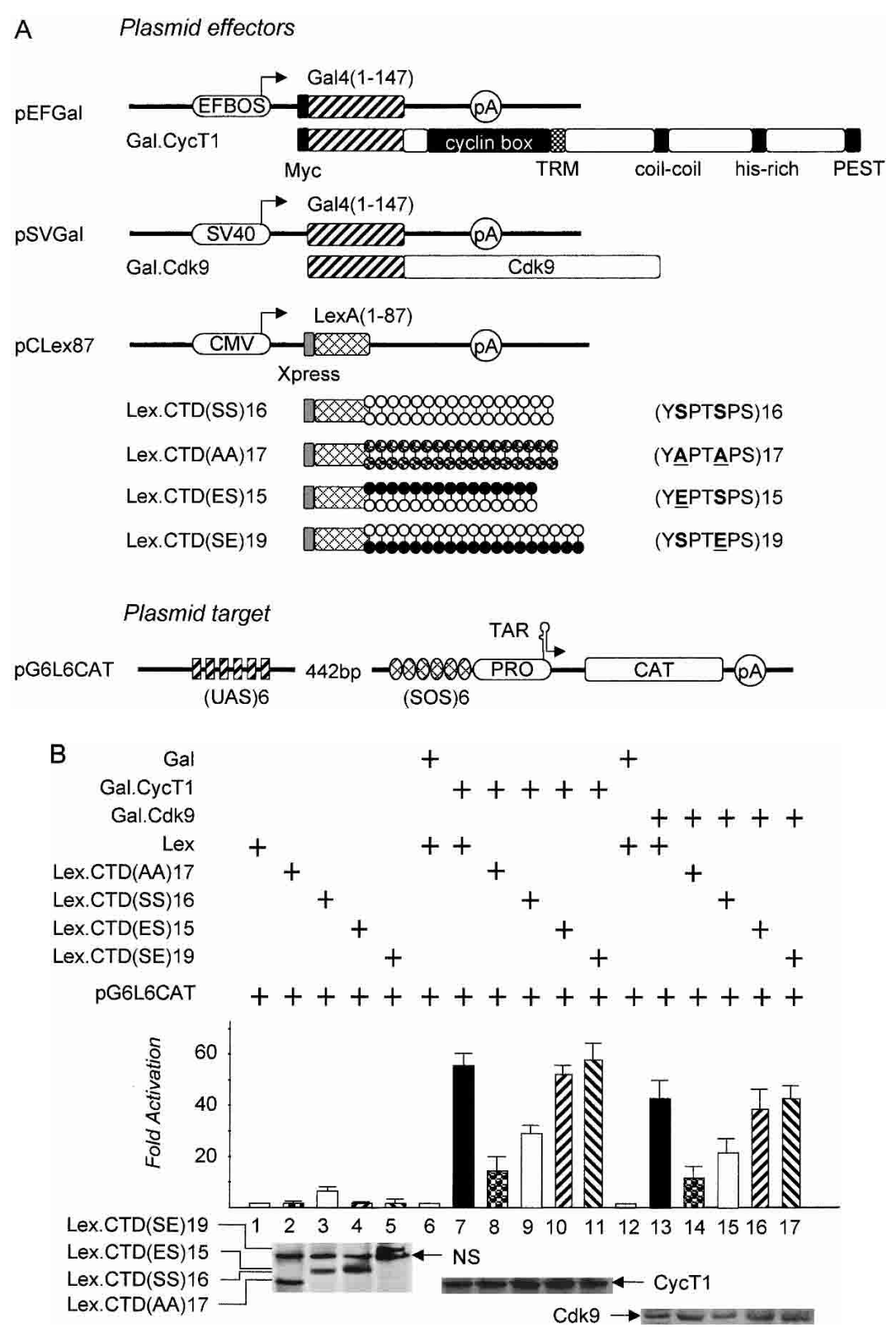

that their overall charge plays an important role in their interactions with CycT1. In this scenario, only the unphosphorylated CTD analogs would bind CycT1. To examine this prediction, we performed GST pull-down assays (Fig. 2). We expressed CycT1 as a GST-fusion protein in Escherichia coli. We transcribed and translated the wild-type Lex.CTD(SS)16 and mutant Lex.CTD(AA)17, Lex.CTD(ES)15 and Lex.CTD(SE)19 chimeras using the rabbit reticulocyte lysate in the presence of ${ }^{35} \mathrm{~S}$-cysteine in vitro (IVT). These proteins were then combined and the bound CTD analogs were eluted from glutathioneSepharose beads and detected by autoradiography. As presented in Figure 2A, the wild-type Lex.CTD(SS)16 and mutant Lex.CTD(AA)17 fusion proteins bound the hybrid GST.CycT1 protein (Fig. 2A, cf. lanes 1,2 and 3,4). Interestingly, only the lower, unphosphorylated wildtype Lex.CTD(SS)16 chimera bound this target (Fig. 2A, lane 4, 2B, lane 2). In contrast, the mutant Lex.CT$\mathrm{D}(\mathrm{ES}) 15$ and LexCTD(SE)19 fusion proteins failed to do so (Fig. 2A, lanes 5-8). Inputs of CTD analogs and GSTfusion proteins were comparable (Fig. 2B). We conclude that only the unphosphorylated, but not phosphorylated CTD analogs bind CycT1. As this binding correlated with their inhibitory effects on P-TEFb, these artificial heptapeptide repeats functioned as a transcriptional repressor. 


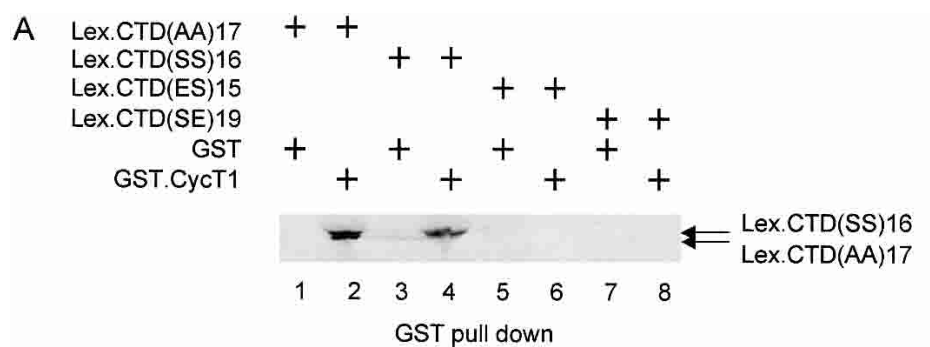

B

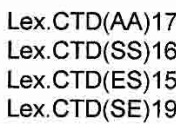

Lex CTD(SE) 19
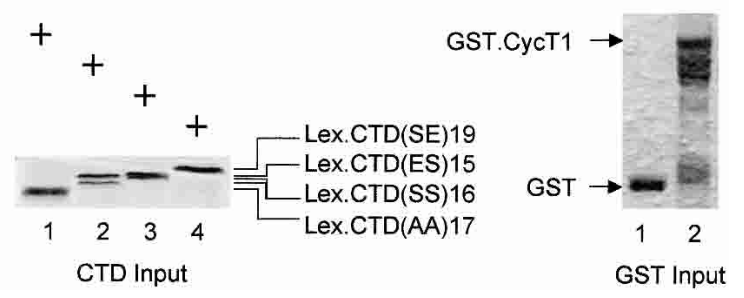

Figure 2. Wild-type and alanine-substituted CTD heptapeptide repeats bind CycT1. (A) GST pulldown. Binding reactions (lanes 1-8) were performed as indicated by plus signs above the autoradiograph. Arrows to the right point to the wild-type Lex.CTD(SS)16 and the mutant Lex.CTD(AA)17 chimeras. (B, Left) The input of Lex.CTD fusion proteins from IVT. Plus signs above the autoradiograph indicate the Lex.CTD fusion proteins. Inputs represent $10 \%$ of the amounts that were used for GST pull-downs. Curved lines to the right point to the Lex.CTD fusion proteins. (Right) The input of GST and GST.CycT1 fusion protein.
Only the wild-type, but not aspartate-substituted and glutamate-substituted heptapeptide repeat in PIE-1 represses transcriptional activation by P-TEFb

To this point, we investigated transcriptional repression by artificial heptapeptide repeats. Thus, we wanted to find a physiological repressor that contains this signature sequence, that is, alanines rather than serines at positions 2 and 5. Just such a heptapeptide repeat, YAPMAPT (from positions 285-291), was found in PIE-1. When tethered to DNA, the repressor domain in PIE-1 containing this heptapeptide repeat inhibited effects of the SV40 enhancer in HeLa cells. Moreover, the change of alanines in YAPMAPT to serines or the introduction of acidic residues (DAQMEQT) abolished this transcriptional repression in vivo (Batchelder et al. 1999). Because the artificial alanine-substituted CTD analog acted as a powerful repressor of P-TEFb (Fig. 1B), we hypothesized that PIE-1 could represent a physiological repressor that would act equivalently and serve as a proof-of-concept. For these experiments, we used the same strategy as presented in Figure 1A, except that instead of CTD analogs, we examined various Lex.PIE-1 fusion proteins for their effects on transcription activated by P-TEFb (Fig. 3A). As with CTD analogs, the wild-type PIE-1 protein (Lex.PIE1), its C terminus (Lex.PIE-1C), mutant serine-substituted [Lex.PIE-1C(YSPMSPT)], and acidic [Lex.PIE1C(DQEQ)] heptapeptide repeats were linked to the LexA DBD (Fig. 3A).

First, we coexpressed pG6L6CAT with Gal.CycT1 and Gal.Cdk9 fusion proteins in Hela cells. Similar to Figure 1B, CycT1 and Cdk9 increased CAT activities 53-fold and 45-fold over basal levels, respectively (Fig. 3B, cf. lanes 1,7 and 2,8). Next, we additionally coexpressed Lex.PIE-1 or Lex.PIE-1C with these effectors and target. As presented in Figure 3B, both Lex.PIE-1 chimeras greatly repressed effects of P-TEFb $(85 \%$ or $80 \%$, respectively; Fig. 3B cf. lanes 2,8 and 3,4 or 9,10). Finally, coexpressed mutant Lex.PIE-1C(STS) and Lex.PIE1C(DQEQ) chimeras decreased this activity by $50 \%$, and not at all, respectively (Fig. 3B, cf. lanes 2,8 and 5,6 or
11,12). The expression levels of LexA fusion proteins were comparable (Fig. 3A, bottom, lanes 3-6). In addition, as the deletion of LexA operator sites (SOS) from pG6L6CAT target plasmid abrogated their effects, the repression of PIE-1 chimeras depended on their recruitment to the promoter (data not shown). Thus, whereas the wild-type Lex.PIE-1C(YAPMAPT) chimera repressed $\mathrm{P}-\mathrm{TEFb}$, the substitution of aspartate and glutamate in the heptapeptide repeat abrogated this effect. We conclude that effects of PIE-1 correlate nicely with those of our CTD analogs. Thus, PIE-1 appears to be a cellular repressor that functions analogously to our artificial CTD analogs.

Wild-type alanine-containing, or the mutant unphosphorylated serine-containing heptapeptide repeats are required for the binding between PIE-1 and $\mathrm{CycT} 1$

To extend the analogy between CTD analogs and PIE-1, we investigated whether PIE-1 also binds CycT1. First, total cell lysates were prepared from COS cells expressing the Flag epitope-tagged PIE-1 protein or the Flag epitope alone and incubated with equivalent amounts of the immobilized GST.CycT1 chimera or GST alone. Bound proteins were eluted and detected with the anti-Flag antibody by Western blotting. As presented in Figure 4A, the Flag.PIE-1 fusion protein bound the GST.CycT1 chimera (Fig. 4A, cf. lanes 1 and 2,3). To analyze the importance of the heptapeptide repeat for this binding, we next performed a GST pull-down assay. Wild-type PIE-1 protein, and mutant PIE-1C, PIE-1C(STS), and PIE1C(DQEQ) Flag epitope-tagged fusion proteins were expressed by IVT. These proteins were then incubated with the same amounts of immobilized GST or GST.CycT1 fusion proteins and the bound PIE-1 chimeras were eluted and detected by autoradiography. Predictably, the wild-type Flag.PIE-1 and mutant Flag.PIE-1C, and Flag.PIE-1C(STS) fusion proteins bound the GST.CycT1 chimera (Fig. 4B, lanes 1-6). In contrast, the mutant 
Zhang et al.

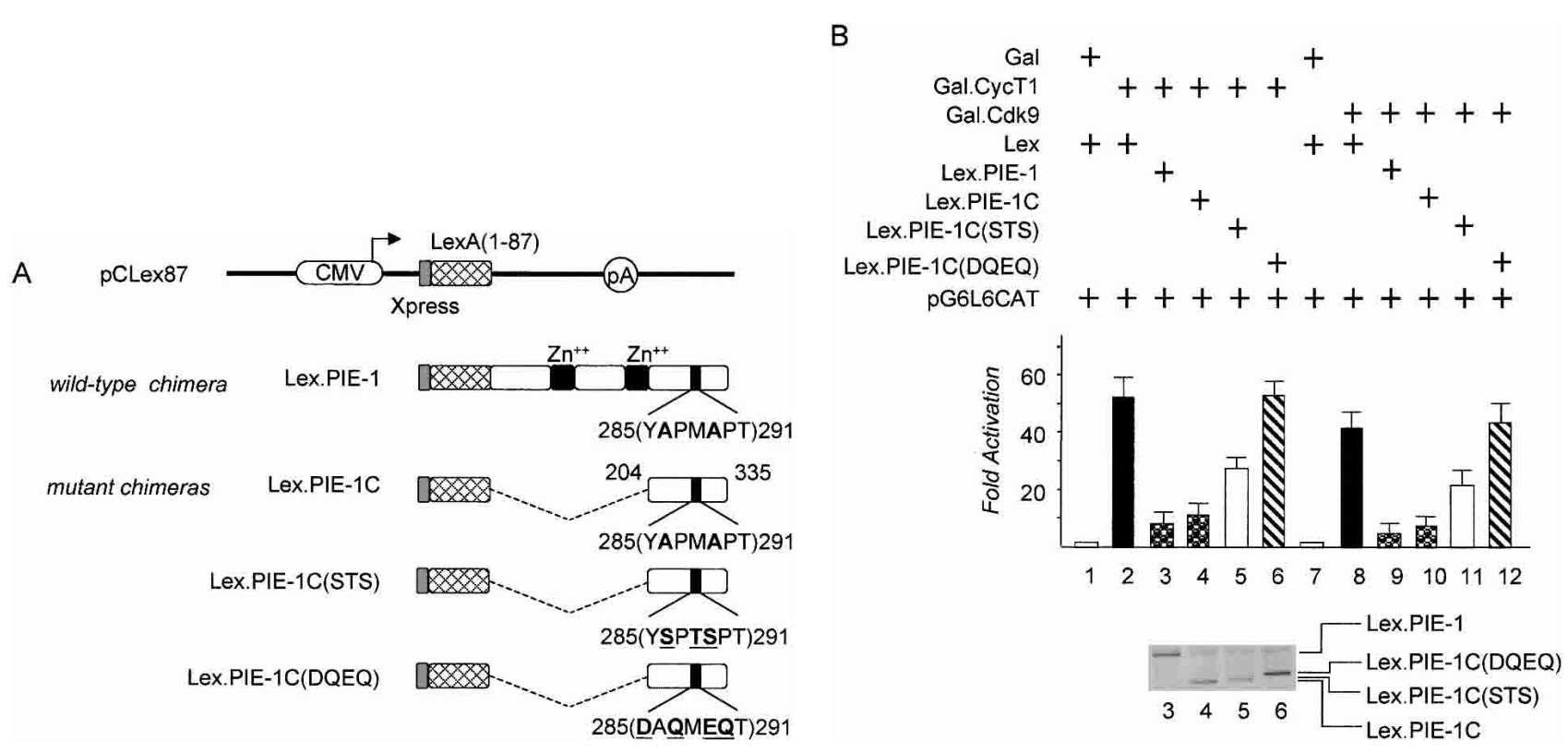

Figure 3. PIE-1 requires the CTD-like repeat to repress transcriptional activation by P-TEFb. (A) Schematic representation of plasmid effectors. The plasmid effector Lex.PIE-1 directed the expression of the wild-type Lex.PIE-1 fusion protein from the CMV promoter. Also presented are two zinc-finger motifs ( $\mathrm{Zn}^{++}$, black boxes) and the CTD-like repeat (CTD, 285-291, black box) in PIE-1. The plasmid effectors Lex.PIE-1C, Lex.PIE-1C(STS), and Lex.PIE-1C(DQEQ) encoded mutant chimeras and expressed the C terminus of PIE-1 (PIE-1C, 204-335, white box) from the CMV promoter. The wild-type and mutant proteins were fused at their $\mathrm{N}$ termini to the LexA DBD [LexA(1-87), cross-hatched box], and the X-press epitope tag (Xpress, gray box). Mutant Lex.PIE-1C, Lex.PIE-1C(STS) and Lex.PIE$1 \mathrm{C}(\mathrm{DQEQ})$ fusion proteins contain depicted heptapeptide sequences. Bold letters within heptapeptides represent wild-type amino acids, whereas underlined bold letters indicate the mutated ones. The arrows indicate the start site of transcription. Encircled pA indicates the polyadenylation site. (B) PIE-1 represses P-TEFb. Hela cells expressed pG6L6CAT (bars 1-12). Proteins that were coexpressed with the plasmid reporter are indicated by plus signs above CAT data. Expression levels of LexA fusion proteins are presented below the CAT data and are indicated by the curved lines.

Flag.PIE-1(DQEQ) fusion protein did not bind the GST.CycT1chimera (Fig. 4B, lanes 7,8). Interestingly, the mutant Flag.PIE-1C(STS) fusion protein migrated as two discrete bands, of which only the lower band bound the
GST.CycT1 chimera. Additionally, the migrations of the mutant Flag.PIE-1(DQEQ) and the upper band of the mutant Flag.PIE-1C(STS) chimeras coincided, thus indicating that acidic and phosphorylated forms of the protein
Figure 4. PIE-1 requires the CTD-like repeat to bind CycT1. (A) PIE-1 binds CycT1. COS cell lysates expressing the Flag epitope-tagged wildtype PIE-1 fusion protein (Flag.PIE-1, lanes 1,2) or the Flag epitope tag alone (Flag, lane 3) were incubated with the GST.CycT1 fusion protein (lanes 1,3) or GST alone (lane 2) as indicated by plus signs above the Western blot. The arrow to the right indicates the presence of Flag.PIE1. $(B)$ PIE-1 binds CycT1 through its CTD-like repeat. (Left) Binding reactions (lanes 1-8) that were performed as indicated by plus signs above the autoradiograph. Arrows to the right point to the bound Flag epitope-tagged PIE-1 proteins. (Right) Inputs of Flag epitope-tagged PIE-1 fusion proteins from IVT as indicated by plus signs above the autoradiograph. Inputs represent $10 \%$ of the amounts that were used for GST pull-downs. The curved lines to the right point to the Flag epitopetagged PIE-1 fusion proteins.
A
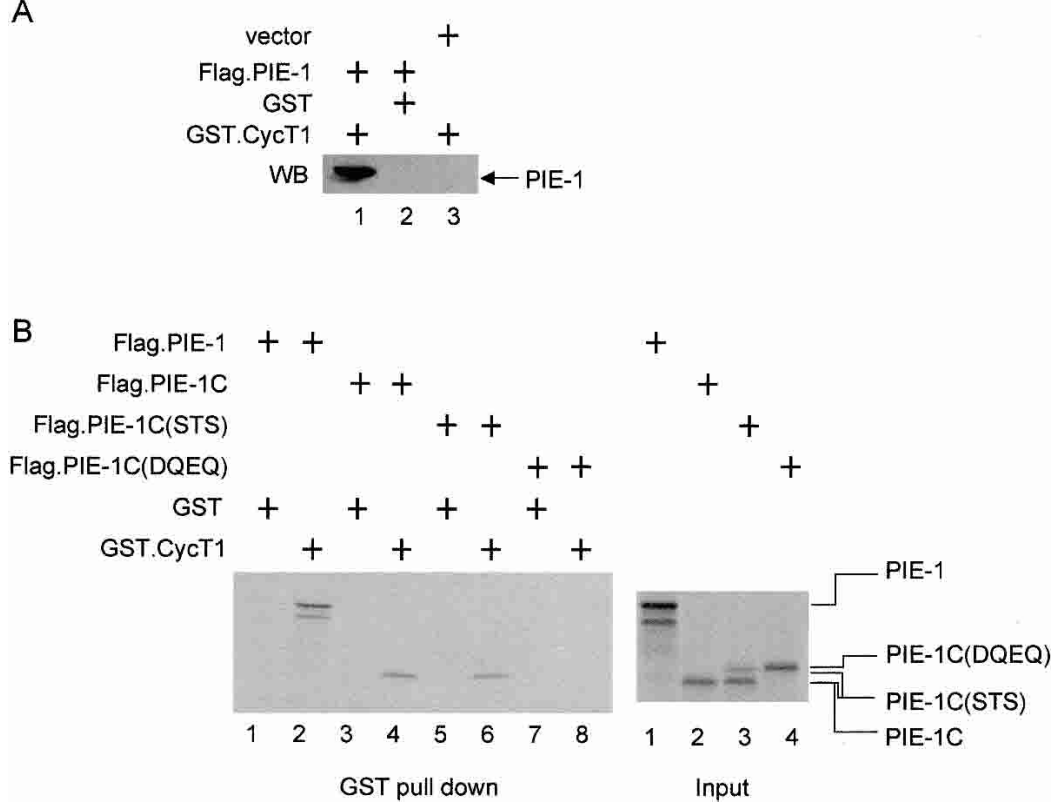
behaved equivalently (Fig. 4B, right, lanes 3,4). Also, the inputs of Flag epitope-tagged PIE-1 fusion proteins were comparable (Fig. 4B, right). Thus, the modification of the heptapeptide repeat in PIE-1 by phosphorylation and/or change to acidic residues resulted in the loss of its binding to CycTl. This situation precisely mirrors data with our CTD analogs (Fig. 2). We conclude that PIE-1 and alanine-substituted heptapeptide repeats bind CycT1, which correlates nicely with the transcriptional repression of P-TEFb.

\section{PIE-1 binds and competes with the CTD for the} binding to the $C$ terminus of CycT1

To investigate which region of CycT1 binds PIE-1, we expressed deletion mutants of CycT1 as GST-fusion proteins in E. coli. The Flag epitope-tagged PIE-1 fusion protein was expressed by IVT (Fig. 5A). These proteins were then combined and analyzed as above. As presented in Figure 5A, lanes 2 and 3, PIE-1 bound the wild-type GST.CycT1(1-726) and mutant GST.CycT1(1-551) fusion proteins but not the mutant GST.CycT1(1-300) chimera that contained two $\mathrm{N}$-terminal cyclin boxes (Fig. $5 \mathrm{~A}$, lane 1).

Intriguingly, the binding pattern between PIE-1 and CycT1 resembled that between the CTD of RNAPII and CycT1 (Taube et al. 2002). Both proteins bound the same region of CycT1 (from positions 300-551), which contains the histidine-rich sequence (from positions 481551). This raised the tantalizing possibility that PIE-1 diverts the histidine-rich sequence in CycT1 away from the transcription complex, thus blocking the interaction between P-TEFb and RNAPII. To examine this possibil-

A

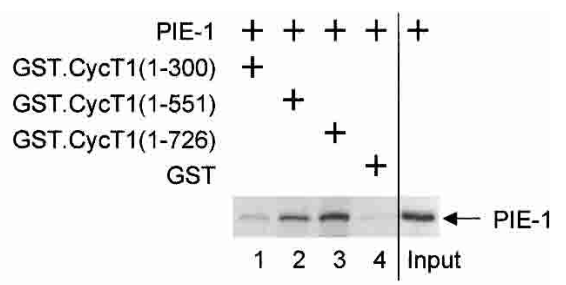

B

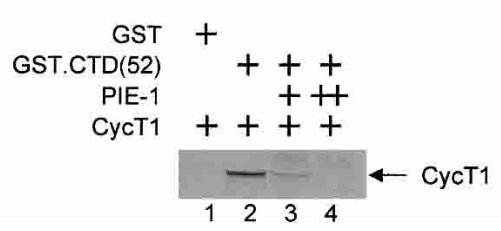

Figure 5. PIE-1 binds and competes with the CTD for the binding to CycT1. (A) The $\mathrm{C}$ terminus of CycT1 binds PIE-1. Binding reactions (lanes 1-4) were performed as indicated by plus signs above the autoradiograph. "Input" lane shows 20\% of the input PIE-1, and lanes 1-4 represent pull-downs. Arrows to the right indicate the presence PIE-1. (B) PIE-1 competes with the CTD for the binding to CycT1. Binding reactions (lanes 1-4) were performed as indicated by plus signs above the autoradiograph. The arrow to the right indicates the presence of CycT1. ity, we performed a competition-binding assay in vitro (Fig. 5B). We expressed Flag epitope-tagged PIE-1 and CycT1 proteins by IVT. Next, the immobilized GST or GST.CTD fusion proteins were incubated with CycT1 alone or together with increasing amounts of PIE-1 and CycT1. Predictably, CycT1 bound the GST.CTD chimera (Fig. 5B, lanes 1,2). Increasing amounts of PIE-1 inhibited this binding (Fig. 5B, lanes 3,4). Thus, by binding the same region in CycT1, PIE-1 blocks the interaction between CycT1 and RNAPII.

\section{The C terminus of PIE-1 represses transcriptional elongation by $P$-TEFb}

To directly demonstrate that PIE-1 inhibits transcriptional elongation by P-TEFb, we performed RNase protection assays (Fig. 6). For these experiments, we used the same plasmid effectors and target as in Figure 3. To independently evaluate levels of initiated and elongated transcripts, we used two different RNA probes. Whereas the promoter-proximal probe measured total levels of transcription, the distal probe measured only levels of elongated transcription. First, we coexpressed pG6L6CAT with the Gal.CycT1 fusion protein in COS cells. In contrast to the control plasmid target, proximal and distal probes gave equivalent signals with the coexpressed Gal.CycT1 chimera (Fig. 6, cf. lanes 1 and 2). In sharp contrast, when we coexpressed the Lex.PIE-1C fusion protein with this effector and target, levels of elongated but not total transcripts were reduced greatly (Fig. 6, cf. lanes 2 and 3). Importantly, whereas the mutant Lex.PIE1 (STS) fusion protein reduced levels of elongated transcripts by $60 \%$, the mutant Lex.PIE-1C(DQEQ) chimera had no effect (Fig. 6, lanes 4,5). The expression of all fusion proteins was comparable (Fig. 3). We conclude that the intact heptapeptide repeat of PIE-1 is critical for inhibiting effects of P-TEFb. Thus, a physiological repressor binds P-TEFb and blocks its ability to promote transcriptional elongation in cells.

\section{Discussion}

In this study, alanine-substituted CTD analogs bound CycT1 and repressed transcriptional effects of P-TEFb. Moreover, PIE-1 behaved identically via its alanine-containing heptapeptide repeat. It also competed for the binding between CycT1 and the CTD. The end result was that P-TEFb could no longer modify the transcription complex for productive elongation. Thus, we propose a new mechanism for blocking eukaryotic gene expression, in which a repressor acts on a rather late step, when RNAPII, PIC, and other components of the transcriptional machinery had already assembled and cleared the promoter.

In our assays, alanine-substituted CTD analogs and the wild-type PIE-1 protein repressed better than the wild-type CTD and the mutant PIE-1 protein with the serine-substituted heptapeptide repeat. Together with the lack of binding between CycT1 and mutant CTD or 
Figure 6. The $\mathrm{C}$ terminus of PIE-1 represses transcriptional elongation by P-TEFb. (Top) RNase protection assay. Proteins that were coexpressed together with pG6L6CAT are indicated above the autoradiograph. Arrows to the right point to protected proximal and distal transcripts. (Bottom) Schematic drawing of pG6L6CAT. The positions of proximal $(-10 /+59)$ and distal $(+342)$ +482 ) antisense RNA probes are presented as black bars.
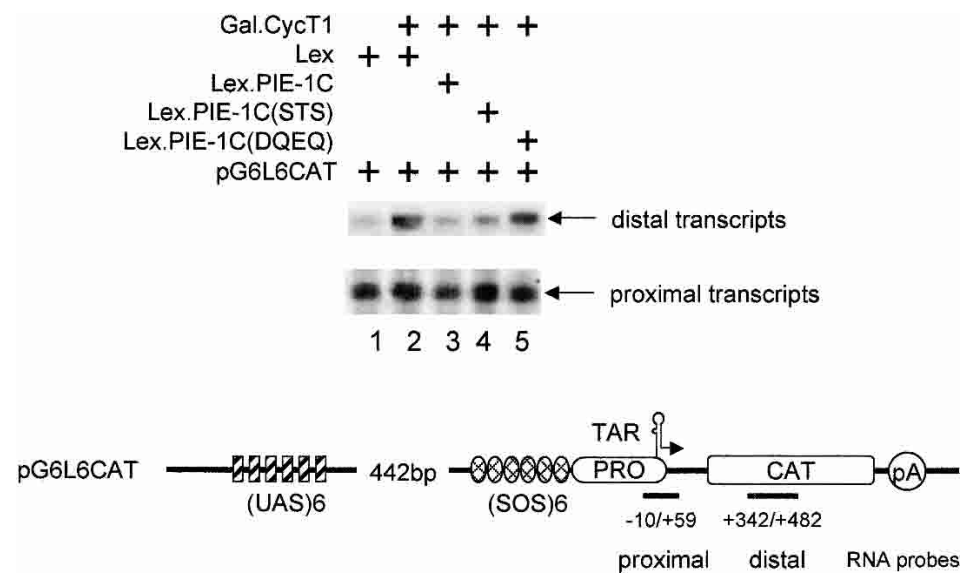

PIE-1 proteins bearing phosphorylated serines or acidic residues, these data indicate that only the unphosphorylated CTD binds and inhibits P-TEFb. Moreover, the alanine-substituted CTD and the wild-type PIE-1 protein behaved as "pseudosubstrates" of Cdk9, which was then unable to phosphorylate the CTD of RNAPII. That the wild-type Lex.CTD(SS)16 chimera had some activity on its own, suggests that it recruited P-TEFb when unphosphorylated and released the complex once phosphorylated. The liberated P-TEFb could then modify other adjacent complexes. This scenario appears more likely than the recruitment of RNAPII, which had been proposed earlier (Seipel et al. 1994), because these effects were on the elongation rather than initiation of transcription.

Although our assays measured effects of P-TEFb and CTD analogs on a linear DNA template, we can extrapolate them to more complex biological systems. First, following heat shock, P-TEFb is also recruited rapidly to sequences, which are several hundred nucleotides upstream of promoters in Drosophila melanogaster (Lis et al. 2000). Second, PIE-1 is a physiological eukaryotic repressor (Batchelder et al. 1999). Third, when placed several kilobases upstream of a strong transcription unit, via its $\mathrm{C}$ terminus and its wild-type, but not mutant heptapeptide repeat, it blocks effects of the SV40 enhancer in HeLa cells (Batchelder et al. 1999). Thus, together with this study, PIE-1 represses transcription when placed 3' and $5^{\prime}$ to activators. Fourth, P-TEFb is recruited by several transcription factors that mediate effects of enhancers. Among these are RelA from NF- $\kappa \mathrm{B}$ (Barboric et al. 2001), CIITA (Kanazawa et al. 2000), c-Myc (Eberhardy and Farnham 2002), and the androgen receptor (Lee et al. 2001). Finally, our work extends the notion proposed by studies investigating the transcriptional regulation of heat-shock genes (Rougvie and Lis 1988) and HIV (Taube et al. 2002), namely, that RNAPII can be fully poised to copy the genome, but unless modified, it waits arrested on the DNA. In this scenario, promoters recruit PIC and position RNAPII correctly at the start sites of genes, whereas enhancers modify RNAPII and N-TEF to allow for productive elongation and cotranscriptional processing.
Importantly, this study addresses the transcriptional repression that occurs rather late in the process of eukaryotic gene expression. To date, a number of studies suggested that gene silencing can be achieved at an earlier stage by means of insulators and boundary elements, some of which faciliate histone deacetylation as well as condensation and/or differential looping of chromatin (Bell et al. 2001). For example, the CCCCTC-binding factor (CTCF), which is highly conserved in vertebrates, binds the chicken $\beta$-globin insulator to block effects of upstream enhancers (Bell et al. 1999, 2001). In this case, changes in chromatin might have separated enhancers from promoters. In D. melanogaster, the suppressor of Hairy-wing [su(Hw)] binds gypsy, a transposable DNA element, to inhibit effects of tissue-specific enhancers during the regulation of the yellow $(\mathrm{y})$ gene expression (Geyer and Corces 1992). Therein, beside effects on chromatin, decoying of activators has been proposed. Additionally, the Groucho/Tup1 corepressors inhibit transcription by recruiting HDACs, thus preventing the PIC assembly (Chen et al. 1999; Watson et al. 2000). In these systems, the promoters may remain unoccupied. Thus, neither the initiation nor the elongation of transcription are observed. Alternatively, a corepressor Tup1 binds the Srb7 subunit of the Mediator /Gromoller and Lehming 2000), thus possibly preventing further stimulation of PIC assembly by a number of activator proteins. In our study, we present evidence for a transcriptional repression at a post-initiation step, in which repressors that resemble the CTD, such as PIE-1, antagonize P-TEFb, thus blocking productive elongation of transcription. Our model is consistent with genetic studies in C. elegans, in which the phosphorylation of serine 2 in the CTD depends on P-TEFb (Shim et al. 2002). Moreover, this phosphorylation is undetectable in embryonic germcell precursors that express PIE-1 (Seydoux and Dunn 1997). Because the phosphorylation of serine 5 in the CTD is present (Seydoux and Dunn 1997) and requires the Mediator and $\mathrm{TF}_{\mathrm{II}} \mathrm{B}$, the initiation of transcription remains intact in these cells (Walker et al. 2001; Shim et al. 2002). Multiple modes of transcriptional repression may have evolved to address specific biological programs. For example, in C. elegans, the phosphorylation 
of serine 2 in the CTD and transcription by RNAPII are detected in somatic cells immediately upon disappearance of PIE-1, indicating that the release of a rapidly reversible block allows for the prompt onset of differentiation program in this organism. Thus, if the function of a repressor is to inhibit transcription transiently, then its noncovalent interactions with a component of the basal transcriptional machinery might be its preferred route of action.

In Figure 7, we present the model of repression addressed in this study. In Figure 7A, we summarize the findings, in which $\mathrm{P}-\mathrm{TEFb}$ activated a strong promoter from positions $5^{\prime}$ and $3^{\prime}$ of the gene (Taube et al. 2002). For simplicity, distal elements are termed enhancers. The histidine-rich sequence in CycT1 binds the CTD of RNAPII from afar and brings Cdk9 to phosphorylate it and other targets, such as N-TEF. Cdk9 prefers the serine at position 2, but can modify the serine at position 5 as well (Zhou et al. 2000). Elongation of transcription ensues. In the presence of a repressor (Fig. 7B), the histidine-rich stretch of CycT1 binds the heptapeptide repeat that cannot be phosphorylated. P-TEFb is held by the repressor and is unable to interact with RNAPII. Thus, the repressor decoys P-TEFb away from the transcription complex. PIC may clear the promoter, but productive elongation of transcription is not observed. Central to this model is the recruitment of P-TEFb after PIC assembly, in this case by enhancers, and the ability of CycT 1 to bind the unphosphorylated CTD of RNAPII. Thus far, PIE-1 supports this model and other eukaryotic repressors of this type will be found. In the meantime, our artificial CTD analogs might become useful tools for eu- karyotic biology. One can envision placing SOS or UAS near transcription units and inducing the expression of these repressors by a variety of approaches to inhibit the transcription of target genes or loci at specific times and in different tissues.

\section{Materials and methods}

\section{Cell culture and cell lines}

Monkey embryonic kidney COS and human Hela cells were maintained in DMEM containing $10 \%$ fetal calf serum, $100 \mathrm{mM}$ L-glutamine, and $50 \mu \mathrm{g}$ each of penicillin and streptomycin per milliliter. All cells were grown at $37^{\circ} \mathrm{C}$ with $5 \% \mathrm{CO}_{2}$.

\section{Plasmid DNAs}

Plasmid target pG6L6CAT was made by inserting two oligonucleotides into the plasmid reporter pHIVSCAT (Lu et al. 1993). The first one contained six modified LexA operator sequences (5'-GTACTGTATGTACATACAGTAC-3') and was inserted into the $X m a I$ site, whereas the second one with six modified Gal4 DNA-binding sequences (5'-CGGAGTACT GTCCTCCGAG-3') was inserted into the KpnI site, which is located $442 \mathrm{bp}$ upstream of LexA operator sites. Plasmids encoding the Gal.CycT1 and Gal.Cdk9 fusion proteins were described previously (Taube et al. 2002). To create pCLex87, a cDNA coding for LexA DNA-binding domain (amino acids 1-87) was inserted into KpnI sites of pcDNA3.1HisB (Invitrogen). Plasmids encoding wild-type Lex.CTD(SS)16, mutant Lex.CTD(AA)17, Lex.CTD(ES)15, and Lex.CTD(SE)19 chimeras were made by inserting amplified PCR fragments of different CTD analogs into the EcoRI and ApaI sites of pCLex87. The
A
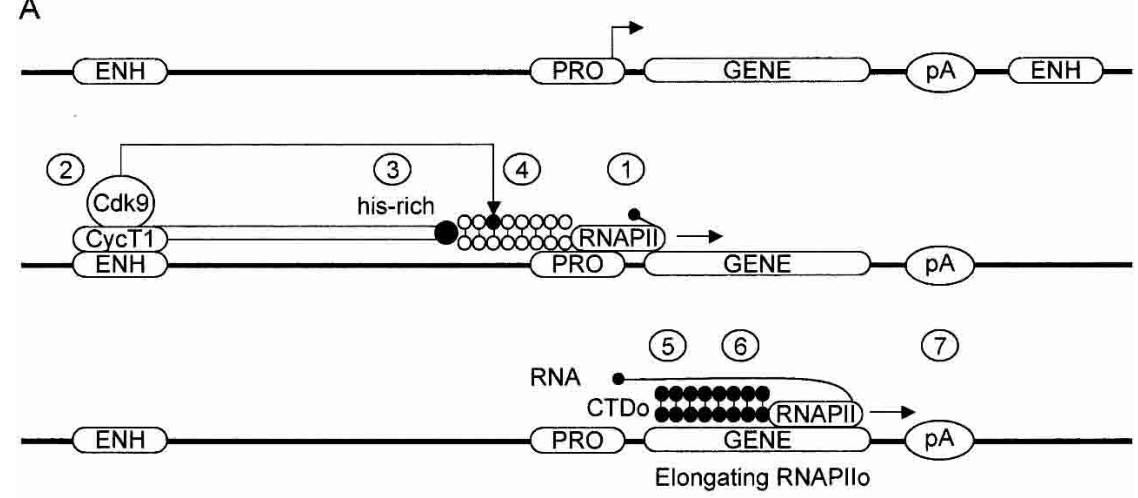

B

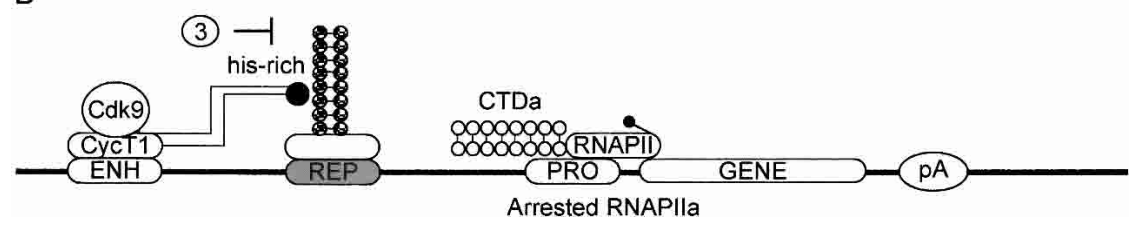

Figure 7. A model of transcriptional repression of P-TEFb. $(A)$ During eukaryotic gene expression, promoters and enhancers play distinct roles. Promoters (PRO) are found at the transcription initiation sites. Enhancers (ENH) are independent of their orientation and distance relative to the RNA start site. Transcription starts with the formation of PIC involving RNAPII recruited to the promoter (step 1). Two rows of balls represent the CTD of RNAPII with serines at positions 2 (top row) and 5 (bottom row). The phosporylated serine is depicted as a black ball. For simplicity, only eight repeats are depicted. P-TEFb is recruited by transcriptional activators, such as, NF-кB, CIITA, Myc, and steroid receptors (step 2). This binding brings P-TEFb into the close proximity of RNAPII, whereupon the histidine-rich stretch in the $\mathrm{C}$ terminus of CycT1 interacts with the unphosphorylated CTD of RNAPII (step 3). Cdk9 phosphorylates the CTD, primarily at serine 2 (step 4 ), facilitating subsequent events that include transcriptional elongation (step 5), cotranscriptional mRNA processing (step 6), and 3' polyadenylation (step 7). CTDo represents the heavily phosphorylated CTD form. (B) In the event of repression of transcriptional elongation, CTD analogs, such as the one found in PIE-1, are recruited to the DNA (REP). They bind the C terminus of CycT1, decoying CycT1 away from the CTD and subsequent phosphorylation of RNAPII and other targets by Cdk9. CTDa represents the unphosphorylated CTD form. Consequently, the transcription complex is stalled near the promoter and is unable to elongate on the gene. 
DNA templates for PCR were from plasmids, pGEXCTD(SS)16, pGEXCTD(AA)17, pGEXCTD(ES)15, and pGEXCTD(SE)19, and were a gift from Dr. Corden (Johns Hopkins, Baltimore, MD). Similarly, plasmids encoding wild-type Lex.PIE-1, mutant Lex.PIE-1C, Lex.PIE-1C(STS), and Lex.PIE-1C(DQEQ) chimeras were made by inserting amplified PCR fragments into the BamHI and ApaI sites of pLex87. The PCR templates are from constructs that encode wild-type and mutant Gal4(1-147).PIE-1 fusion proteins, which were described previously (Batchelder et al. 1999). To create plasmids that encode Flag-tagged PIE-1, PIE1C, PIE-1C(STS), and PIE-1C(DQEQ) proteins, a Flag-epitope sequence was incorporated into the $5^{\prime}$ primer. PCR fragments amplified from templates mentioned previously were inserted into HindIII and BamHI sites of the pcDNA3.1Hygro expression vector. Plasmids pMTX-89 and pMTX-147 were gifts from Jonathan Karn and were described previously (West and Karn 1999|. The constructs that encode wild-type GST.CycT1 and GST.CTD(52) fusion proteins were described previously (Taube et al. 2002).

\section{Transient transfection and CAT assays}

Hela cells were cotransfected with pG6L6CAT $(0.4 \mu \mathrm{g})$ and different plasmid effectors including both agonists and antagonists (total $1.6 \mu \mathrm{g}$ ) with Lipofectamine according to the manufacturer's instructions (GIBCO-BRL). The ratio of agonists (Gal.CycT1 or Gal.Cdk9) versus antagonists (LexA fusion proteins) was 1:1. All transfections were balanced to the total $2.0 \mu \mathrm{g}$ of DNA with the appropriate empty vectors. At $48 \mathrm{~h}$ after transfection, cells were lysed in the lysis buffer $(0.1 \%$ TritonX-100, 0.25 M Tris$\mathrm{HCl}$ at $\mathrm{pH}$ 7.5). Chloramphenicol acetyltransferase (CAT) enzymatic assays were performed as described (Fujinaga et al. 1998). The activity of the reporter plasmid alone is given as one. Data are representative of three independent transfections, which were performed in duplicates. Error bars give standard errors of the mean.

\section{In vitro binding and in vitro competition assays}

A total of $3 \mu \mathrm{g}$ of the plasmid encoding Flag.PIE-1 or the empty plasmid vector was transfected into COS cells as described above. To prepare total cell lysates, cells were resuspended in the lysis buffer [1\% NP40, $10 \mathrm{mM}$ Tris at $\mathrm{pH} 7.4,150 \mathrm{mM}$ $\mathrm{NaCl}, 2 \mathrm{mM}$ EDTA, $1 \mathrm{mM}$ PMSF, $0.1 \%$ protease inhibitor cocktail (Sigma)] at $4^{\circ} \mathrm{C}$, with gentle agitation for $30 \mathrm{~min}$. These cell lysates were incubated with equal amounts of GST or GST.CycT1 fusion proteins bound to glutathione-conjugated sepharose beads at $4^{\circ} \mathrm{C}$ for $2 \mathrm{~h}$. After the binding reaction, the beads were washed four times with the lysis buffer, and the bound proteins were eluted by boiling in the SDS sample buffer, resolved by SDS-PAGE on a $10 \%$ gel and revealed by Western blotting. For GST pull-down assays, the wild-type and mutant LexA fusion proteins and Flag-epitope tagged PIE-1 proteins were transcribed and translated (IVT) in the presence of ${ }^{35} \mathrm{~S}$ labeled cysteine in vitro using the TNT T7-coupled rabbit reticulocyte lysate system as instructed by the manufacturer (Promega). GST and GST.CycT1 wild-type and mutant fusion proteins were expressed in E. coli BL21(DE3)pLysS competent cells (Novagen), purified, and bound to glutathione-conjugated sepharose beads as described (Kanazawa et al. 2000). A total of $20 \mu \mathrm{g}$ of each GST or GST fusion protein was incubated with $20 \mu \mathrm{L}$ of ${ }^{35}$ S-labeled LexA fusion proteins or Flag-epitope tagged PIE-1 fusion proteins in $300 \mu \mathrm{L}$ of binding buffer $(20 \mathrm{mM}$ HEPES at $\mathrm{pH}$ 7.9, 1\% Triton X-100, 20 mM DTT, 5 mM EDTA, 0.5\% BSA, and $100 \mathrm{mM} \mathrm{KCl}$ ) at $4{ }^{\circ} \mathrm{C}$ with gentle agitation for $2 \mathrm{~h}$. After the binding reaction, the beads were washed four times with the binding buffer, and the bound proteins were eluted by boiling in the SDS sample buffer, resolved by SDS-PAGE on a $12.5 \%$ gel, which was dried and analyzed by autoradiography. To conduct the binding competition assay, $15 \mu \mathrm{L}$ or $30 \mu \mathrm{L}$ of IVT PIE-1 was incubated with $15 \mu \mathrm{L}$ IVT CycT1 (from pCNDA3.1HACycT1) at $4^{\circ} \mathrm{C}$ for $2 \mathrm{~h}$, then CycT1 alone, and the mixtures of PIE- 1 and CycT1 were incubated with equal amounts of GST or GST.CTD(52) fusion protein for an additional $2 \mathrm{~h}$. The bound proteins were revealed by autoradiography as described above.

\section{Immunoreagents and Western blot analysis}

To detect Gal.CycT1 and Gal.Cdk9 fusion proteins, rabbit polyclonal anti-Myc (sc-789) and mouse monoclonal anti-Cdk9 (sc13130) antibodies were used, respectively, that were obtained from Santa Cruz Biotechnology. To detect Flag.PIE-1 fusion proteins, mouse monoclonal anti-Flag M2 antibody (F3165, Sigma) was used. To detect LexA fusion proteins, mouse monoclonal anti-X-press antibody (46-0528) was used, which was obtained from Invitrogen. Western blotting was performed according to standard protocols.

\section{Ribonuclease protection assays}

COS cells $\left(6 \times 10^{5}\right.$ cells per plate) were transfected with pG6L6CAT ( $2 \mu \mathrm{g})$, plasmids encoding Gal.CycT1 $(5 \mu \mathrm{g})$ and indicated LexA fusion proteins $(3 \mu \mathrm{g})$, or the corresponding amounts of empty plasmid vectors. Cells were harvested $24 \mathrm{~h}$ post-transfection, and total RNA was extracted using TRIzol reagent (GIBCO-BRL). Template DNAs for the preparation of antisense probes were generated as described (West and Karn 1999|. Antisense probes were prepared using MAXIscript T3 Kit (Ambion) according to manufacturer's recommendations. The ribonuclease protection assays were performed on $20 \mu \mathrm{g}$ of total RNA and 20,000 c.p.m/10 $\mu$ g of antisense probes using the RPA III Ribonuclease Protection Assay Kit (Ambion) according to manufacturer's recommendations.

\section{Acknowledgments}

We thank Dan Irwin, Leonore Wigger, and Paula Zupanc-Ecimovic for secretarial assistance; David Price and Jeffrey Corden for help and reagents, and the members of our laboratory for helpful discussions. F.Z. is the recipient of the 2002 amFAR fellowship award (70576-31-RFT). M.B. was partially supported by a fellowship from the Ministry of Education, Science and Sport, Republic of Slovenia. This work was supported by grants from the American Foundation for AIDS research (amFAR), NIH and UARP. T.K.B. is supported by grants from the March of Dimes.

The publication costs of this article were defrayed in part by payment of page charges. This article must therefore be hereby marked "advertisement" in accordance with 18 USC section 1734 solely to indicate this fact.

\section{References}

Barboric, M., Nissen, R.M., Kanazawa, S., Jabrane-Ferrat, N., and Peterlin, B.M. 2001. NF-кB binds P-TEFb to stimulate transcriptional elongation by RNA polymerase II. 2001. Mol. Cell 8: 327-337.

Bartolomei, M.S., Halden, N.F., Cullen, C.R., and Corden, J.L. 1988. Genetic analysis of the repetitive carboxyl-terminal domain of the largest subunit of mouse RNA polymerase II. Mol. Cell. Biol. 8: 330-339. 
Batchelder, C., Dunn, M.A., Choy, B., Suh, Y., Cassie, C., Shim, E.Y., Shin, T.H., Mello, C., Seydoux, G., and Blackwell, T.K. 1999. Transcriptional repression by the Caenorhabditis elegans germ-line protein PIE-1. Genes \& Dev. 13: 202-212.

Bell, A.C., West, A.G., and Felsenfeld, G. 1999. The protein CTCF is required for the enhancer blocking activity of vertebrate insulators. Cell 98: 387-396.

. 2001. Insulators and boundaries: Versatile regulatory elements in the eukaryotic genome. Science 291: 447-450.

Blackwood, E.M. and Kadonaga, J.T. 1998. Going the distance: A current view of enhancer action. Science 281: 61-63.

Chao, S.H., Fujinaga, K., Marion, J.E., Taube, R., Sausville, E.A., Senderowicz, A.M., Peterlin, B.M., and Price, D.H. 2000. Flavopiridol inhibits $\mathrm{P}-\mathrm{TEFb}$ and blocks HIV-1 replication. I. Biol. Chem. 275: 28345-28348.

Chen, G., Fernandez, J., Mische, S., and Courey, A.J. 1999. A functional interaction between the histone deacetylase Rpd3 and the corepressor groucho in Drosophila development. Genes \& Dev. 13: 2218-2230.

Cho, H., Kim, T.K., Mancebo, H., Lane, W.S., Flores, O., and Reinberg, D. 1999. A protein phosphatase functions to recycle RNA polymerase II. Genes \& Dev. 13: 1540-1552.

Dahmus, M.E. 1996. Reversible phosphorylation of the C-terminal domain of RNA polymerase II. I. Biol. Chem. 271: 19009-19012.

Eberhardy, S.R. and Farnham, P.J. 2002. Myc recruits P-TEFb to mediate the final step in the transcriptional activation of the cad promoter. J. Biol. Chem. 277: 40156-40162.

Fujinaga, K., Cujec, T.P., Peng, J., Garriga, J., Price, D.H., Grana, X., and Peterlin, B.M. 1998. The ability of positive transcription elongation factor B to transactivate human immunodeficiency virus transcription depends on a functional kinase domain, cyclin T1, and Tat. J. Virol. 72: 7154-7159.

Garber, M.E., Mayall, T.P., Suess, E.M., Meisenhelder, J., Thompson, N.E., and Jones, K.A. 2000. CDK9 autophosphorylation regulates high-affinity binding of the human immunodeficiency virus type 1 tat-P-TEFb complex to TAR RNA. Mol. Cell. Biol. 20: 6958-6969.

Geyer, P.K. and Corces, V.G. 1992. DNA position-specific repression of transcription by a Drosophila zinc finger protein. Genes \& Dev. 6: 1865-1873.

Goldmark, J.P., Fazzio, T.G., Estep, P.W., Church, G.M., and Tsukiyama, T. 2000. The Isw2 chromatin remodeling complex represses early meiotic genes upon recruitment by Ume6p. Cell 103: 423-433.

Gromoller, A. and Lehming, N. 2000. Srb7p is a physical and physiological target of Tup1p. EMBO J. 19: 6845-6852.

Guzman, E. and Lis, J.T. 1999. Transcription factor TFIIH is required for promoter melting in vivo. Mol. Cell. Biol. 19: $5652-5658$

Ho, C.K. and Shuman, S. 1999. Distinct roles for CTD Ser-2 and Ser-5 phosphorylation in the recruitment and allosteric activation of mammalian mRNA capping enzyme. Mol. Cell 3: 405-411.

Ivanov, D., Kwak, Y.T., Guo, J., and Gaynor, R.B. 2000. Domains in the SPT5 protein that modulate its transcriptional regulatory properties. Mol. Cell. Biol. 20: 2970-2983.

Kanazawa, S., Okamoto, T., and Peterlin, B.M. 2000. Tat competes with CIITA for the binding to P-TEFb and blocks the expression of MHC class II genes in HIV infection. Immunity 12: 61-70.

Khochbin, S., Verdel, A., Lemercier, C., and Seigneurin-Berny, D. 2001. Functional significance of histone deacetylase diversity. Curr. Opin. Genet. Dev. 11: 162-166.

Komarnitsky, P., Cho, E.J., and Buratowski, S. 2000. Different phosphorylated forms of RNA polymerase II and associated
mRNA processing factors during transcription. Genes \& Dev. 14: 2452-2460.

Langst, G. and Becker, P.B. 2001. Nucleosome mobilization and positioning by ISWI-containing chromatin-remodeling factors. J. Cell. Sci. 114: 2561-2568.

Lee, D.K., Duan, H.O., and Chang, C. 2001. Androgen receptor interacts with the positive elongation factor P-TEFb and enhances the efficiency of transcriptional elongation. J. Biol. Chem. 276: 9978-9984.

Lemon, B. and Tjian, R. 2000. Orchestrated response: A symphony of transcription factors for gene control. Genes \& Dev. 14: 2551-2569.

Lis, J.T., Mason, P., Peng, J., Price, D.H., and Werner, J. 2000. $\mathrm{P}-\mathrm{TEFb}$ kinase recruitment and function at heat shock loci. Genes \& Dev. 14: 792-803.

Lu, H., Zawel, L., Fisher, L., Egly, J.M., and Reinberg, D. 1992. Human general transcription factor IIH phosphorylates the C-terminal domain of RNA polymerase II. Nature 358: 641645.

Lu, X., Welsh, T.M., and Peterlin, B.M. 1993. The human immunodeficiency virus type 1 long terminal repeat specifies two different transcription complexes, only one of which is regulated by Tat. J. Virol. 67: 1752-1760.

Marshall, N.F., Peng, J., Xie, Z., and Price, D.H. 1996. Control of RNA polymerase II elongation potential by a novel carboxylterminal domain kinase. J. Biol. Chem. 271: 27176-27183.

McCracken, S., Fong, N., Yankulov, K., Ballantyne, S., Pan, G.H., Greenblatt, J., Patterson, S.D., Wickens, M., and Bentley, D.L. 1997. The C-terminal domain of RNA polymerase II couples messenger RNA processing to transcription. $\mathrm{Na}$ ture 385: 357-361.

Mello, C.C., Draper, B.W., Krause, M., Weintraub, H., and Priess, J.R. 1992. The pie-1 and mex-1 genes and maternal control of blastomere identity in early C. elegans embryos. Cell 70: 163-176.

Mello, C.C., Schubert, C., Draper, B., Zhang, W., Lobel, R., and Priess, J.R. 1996. The PIE-1 protein and germline specification in C. elegans embryos. Nature 382: 710-712.

Mortillaro, M.J., Blencowe, B.J., Wei, X.Y., Nakayasu, H., Du, L., Warren, S.L., Sharp, P.A., and Berezney, R. 1996. A hyperphosphorylated form of the large subunit of RNA polymerase II is associated with splicing complexes and the nuclear matrix. Proc. Nat1. Acad. Sci. 93: 8253-8257.

Myers, L.C. and Kornberg, R.D. 2000. Mediator of transcriptional regulation. Annu. Rev. Biochem. 69: 729-749.

Orphanides, G. and Reinberg, D. 2002. A unified theory of gene expression. Cell 108: 439-451.

Orphanides, G., Lagrange, T., and Reinberg, D. 1996. The general transcription factors of RNA polymerase II. Genes \& Dev. 10: 2657-2683.

Peng, J., Zhu, Y., Milton, J.T., and Price, D.H. 1998. Identification of multiple cyclin subunits of human P-TEFb. Genes \& Dev. 12: 755-762.

Prelich, G. 2002. RNA Polymerase II carboxyl-terminal domain kinases: Emerging clues to their function. Euk. Cell 1: 153162.

Price, D.H. 2000. P-TEFb, a cyclin-dependent kinase controlling elongation by RNA polymerase II. Mol. Cell. Biol. 20: 26292634.

Rougvie, A.E. and Lis, J.T. 1988. The RNA polymerase II molecule at the $5^{\prime}$ end of the uninduced hsp70 gene of D. melanogaster is transcriptionally engaged. Cell 54: 795-804.

Seipel, K., Georgiev, O., Gerber, H.P., and Schaffner, W. 1994. Basal components of the transcription apparatus (RNA polymerase II, TATA-binding protein) contain activation domains: is the repetitive C-terminal domain (CTD) of RNA 


\section{Zhang et al.}

polymerase II a 'portable enhancer domain'? Mol. Reprod. Dev. 39: 215-225.

Seydoux, G. and Dunn, M.A. 1997. Transcriptionally repressed germ cells lack a subpopulation of phosphorylated RNA polymerase II in early embryos of Caenorhabditis elegans and Drosophila melanogaster. Development 124: 2191-2201.

Seydoux, G., Mello, C.C., Pettitt, J., Wood, W.B., Priess, J.R., and Fire, A. 1996. Repression of gene expression in the embryonic germ lineage of C. elegans. Nature 382: 713-716.

Shim, E.Y., Walker, A.K., Shi, Y., and Blackwell, T.K. 2002. CDK-9/cyclin $\mathrm{T}(\mathrm{P}-\mathrm{TEFb})$ is required in two postinitiation pathways for transcription in the C. elegans embryo. Genes \& Dev. 16: 2135-2146.

Struhl, K. 1998. Histone acetylation and transcriptional regulatory mechanisms. Genes \& Dev. 12: 599-606.

Taube, R., Lin, X., Irwin, D., Fujinaga, K., and Peterlin, B.M. 2002. Interaction between P-TEFb and the C-terminal domain of RNA polymerase II activates transcriptional elongation from sites upstream or downstream of target genes. Mol. Cell. Biol. 22: 321-331.

Wada, T., Takagi, T., Yamaguchi, Y., Ferdous, A., Imai, T., Hirose, S., Sugimoto, S., Yano, K., Hartzog, G.A., Winston, F., et al. 1998. DSIF, a novel transcription elongation factor that regulates RNA polymerase II processivity, is composed of human Spt4 and Spt5 homologs. Genes \& Dev. 12: 343-356.

Walker, A.K., Rothman, J.H., Shi, Y., and Blackwell, T.K. 2001. Distinct requirements for C.elegans TAF(II)s in early embryonic transcription. EMBO I. 20: 5269-5279.

Watson, A.D., Edmondson, D.G., Bone, J.R., Mukai, Y., Yu, Y., Du, W., Stillman, D.J., and Roth, S.Y. 2000. Ssn6-Tup1 interacts with class I histone deacetylases required for repression. Genes \& Dev. 14: 2737-2744.

West, M.J. and Karn, J. 1999. Stimulation of Tat-associated kinase-independent transcriptional elongation from the human immunodeficiency virus type- 1 long terminal repeat by a cellular enhancer. EMBO J. 18: 1378-1386.

Yamaguchi, Y., Takagi, T., Wada, T., Yano, K., Furuya, A., Sugimoto, S., Hasegawa, J., and Handa, H. 1999. NELF, a multisubunit complex containing RD, cooperates with DSIF to repress RNA polymerase II elongation. Cell 97: 41-51.

Zawel, L. and Reinberg, D. 1995. Common themes in assembly and function of eukaryotic transcription complexes. Annu. Rev. Biochem. 64: 533-561.

Zhou, M., Halanski, M.A., Radonovich, M.F., Kashanchi, F., Peng, J., Price, D.H., and Brady, J.N. 2000. Tat modifies the activity of CDK9 to phosphorylate serine 5 of the RNA polymerase II carboxyl-terminal domain during human immunodeficiency virus type 1 transcription. Mol. Cell. Biol. 20: 5077-5086. 


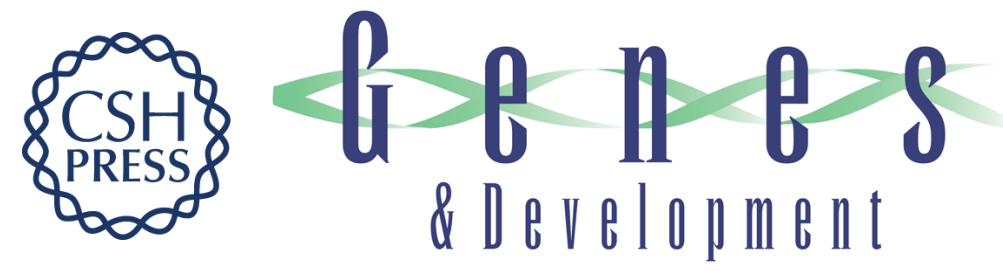

\section{A model of repression: CTD analogs and PIE-1 inhibit transcriptional elongation by P-TEFb}

Fan Zhang, Matjaz Barboric, T. Keith Blackwell, et al.

Genes Dev. 2003, 17:

Access the most recent version at doi:10.1101/gad.1068203

\section{Related Content}

References

License

Email Alerting Service

\section{Repression by Imitation}

Sci. STKE April , 2003 2003: tw126-TW126

This article cites 50 articles, 33 of which can be accessed free at: http://genesdev.cshlp.org/content/17/6/748.full.htmI\#ref-list-1

Articles cited in:

http://genesdev.cshlp.org/content/17/6/748.full.html\#related-urls

Receive free email alerts when new articles cite this article - sign up in the box at the top right corner of the article or click here.

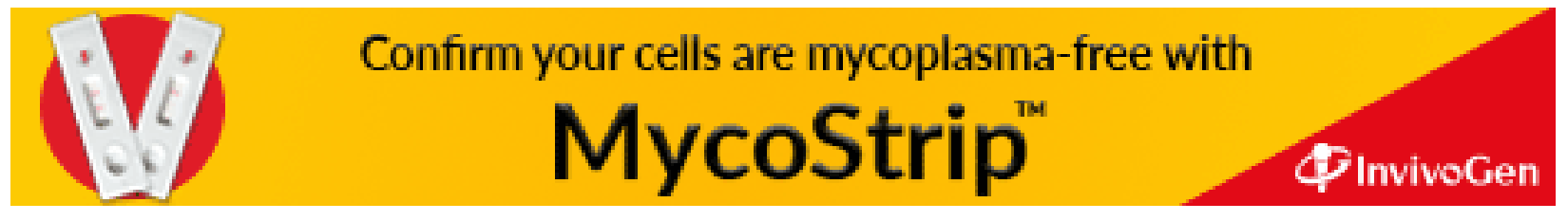

